\title{
Natural convection in a shallow cavity with differentially heated end walls. Part 2. Numerical solutions
}

\author{
By D. E. CORMACK, L. G. LEAL AND J. H. SEINFELD \\ Chemical Engineering, California Institute of Technology, Pasadena
}

(Received 23 March 1973 and in revised form 15 February 1974)

Numerical solutions of the full Navier-Stokes equations are obtained for the problem of natural convection in closed cavities of small aspect ratio with differentially heated end walls. These solutions cover the parameter range $\operatorname{Pr}=6.983,10 \leqslant G r \leqslant 2 \times 10^{4}$ and $0.05 \leqslant A \leqslant 1$. A comparison with the asymptotic theory of part 1 shows excellent agreement between the analytical and numerical solutions provided that $A \lesssim 0.1$ and $G r^{2} A^{3} P r^{2} \lesssim 10^{5}$. In addition, the numerical solutions demonstrate the transition between the shallow-cavity limit of part 1 and the boundary-layer limit; $A$ fixed, $G r \rightarrow \infty$.

\section{Introduction}

This paper reports numerical solutions of the full Navier-Stokes equations, subject to the Boussinesq approximation, for buoyancy-driven convection in a shallow rectangular cavity of height $h$ and length $l(h \ll l)$ with insulated top and bottom, and differentially heated end walls. In part 1, hereafter denoted as I†, Cormack, Leal \& Imberger (1974) presented an asymptotic solution to the problem, valid in the limit as the cavity aspect ratio $A \equiv h / l \rightarrow 0$, for fixed, though arbitrary, values of the Grashof and Prandtl numbers $G r$ and $P r$. It was shown that the flow structure in this limit consists of two distinct regimes: a parallel flow in the central core and a non-parallel flow which is confined to within an $O(h)$ distance of the end walls. The present investigation considers the domain of small but finite $A$ with $G r$ ranging from 10 to $2 \times 10^{4}$. Strong evidence is found to support the asymptotic theory of I. More important, however, the numerical solutions also illustrate the role of $G r$ in establishing the flow structure when $A$ is small, but not vanishingly so. In particular, the transition from the parallel flow regime of I to the boundary-layer limit of Gill (1966) is clearly demonstrated.

Numerical investigations of buoyancy-induced convection in rectangular cavities with differentially heated side walls have been numerous. The first extensive investigation was contributed by Wilkes \& Churchill (1966), who studied the steady-state and transient fluid behaviour for $G r<10^{5}$ and aspect ratios of 1,2 and 3 . However, a more thorough numerical study was reported

$\dagger$ Equations and figures from $I$ will be denoted as IX, where $X$ refers to the original equation or figure number. 
almost simultaneously by Elder (1966), which encompassed the conductiondominated regime of Batchelor's (1954) theoretical analysis, as well as Rayleigh numbers $(R a=G r P r)$ up to $10^{5}$, for aspect ratios varying from 1 to 4 . More recent investigations have been reported by De Vahl Davis (1968) and Newell $\&$ Schmidt (1970). All of these studies have dealt primarily with the dependence of the flow structure and overall Nusselt number on Rayleigh number for $A \geqslant 1$ and large $R a$. The chief qualitative feature evident from these studies, and of interest in the present context, is the existence of end-wall boundary layers, and the corresponding lack of any appreciable horizontal temperature gradients in the core for large values of $R a$ (or $G r$ ).

Most recently Quon (1972) studied the effect of changing the dynamical boundary conditions for large Rayleigh numbers, $R a>10^{5}$, and $A=1$. Changes in the horizontal boundary conditions from free shear to no slip were found to have little influence on the main flow. Furthermore, for one of the cases, the basic assumptions of Gill's (1966) boundary-layer analysis were apparently satisfied. However, Quon's solutions show only modest quantitative agreement with Gill's approximate analysis of the boundary-layer model. On the other hand, his results are in good agreement with the experiments of Elder (1965).

It is surprising that the previous numerical work has been concerned exclusively with aspect ratios $A \geqslant 1$, in spite of the frequent relevance of small aspect ratios to problems of environmental fluid mechanics (see I). In this respect, the present study not only represents a substantial departure from the previous work, but provides a base from which more detailed investigations of the small aspect ratio problem may be undertaken.

\section{Formulation of the numerical problem}

We consider here an enclosed cavity of aspect ratio $A \leqslant 1$, which is filled with a Newtonian fluid. The top and bottom of the cavity are rigid no-slip boundaries which are perfectly insulating, while the side walls are similarly rigid and noslip, but are maintained at different uniform temperatures $T_{h}$ and $T_{c}$. A schematic diagram of the system was presented in figure I1. The governing equations, non-dimensionalized and subject to the Boussinesq approximation, were shown in I to be

and

$$
\begin{gathered}
G r A^{2}\left(\frac{\partial \omega}{\partial t}+\frac{\partial(\omega, \psi)}{\partial(x, y)}\right)=A \nabla^{2} \omega+\frac{\partial \theta}{\partial x} \\
\nabla^{2} \psi=-\omega
\end{gathered}
$$

Here, $\psi$ is the stream function defined in terms of the horizontal and vertical velocity components by $u=\partial \psi / \partial y, \quad v=-\partial \psi / \partial x$,

$\omega$ is the vorticity and $\theta$ is the dimensionless temperature. The relevant boundary conditions are

$$
\left.\begin{array}{l}
\psi=\partial \psi / \partial x=0, \quad \theta=x A \quad \text { on } \quad x=0,1 / A \\
\psi=\partial \psi / \partial y=0, \quad \partial \theta / \partial y=0 \quad \text { on } \quad y=0,1
\end{array}\right\}
$$


In the remainder of this paper we consider the solution of (1)-(3) subject to the conditions (4) using a finite-difference numerical scheme. It is well known that such a treatment introduces errors that depend explicitly on the grid spacing. To minimize this truncation error, it is desirable to use the smallest grid spacing possible throughout the domain of integration. However, since the computation time increases markedly as the number of grid points is increased, one must reach a compromise between the accuracy of the solution and the computation time necessary to reach that solution.

Square cavities have received thorough consideration in this regard. Elder (1966) found that solutions obtained with an $11 \times 11$ grid were qualitatively acceptable, that a marked improvement occurred when the mesh size was halved to $21 \times 21$, but that little further change resulted when a finer mesh was used. The idea of using a scaled grid to reduce the number of mesh points was introduced by Newell \& Schmidt (1970). In this scheme, the smallest grid spacing is maintained in the wall regions of the cavity, where gradients of the field variables are largest, whereas a coarser grid is used to yield comparable accuracy in the central region of the cavity, where gradients are small. Newell \& Schmidt varied the grid spacing in their calculations by applying a nonlinear (polynomial) co-ordinate transformation to (1)-(3), which were then finite differenced using a uniform square mesh. An alternative is to impose the graded mesh directly on the original equations (1)-(3).

The scaled-grid approach of Newell \& Schmidt was adopted for the present work. Several different transformations including the Newell-Schmidt polynomials were tried, as well as various mesh sizes in the transformed planes. For square cavities the most satisfactory choices appeared to be the sine transformations

$$
\begin{gathered}
x=\frac{1}{2 A}\left[1+\frac{\sin X}{\sin 0 \cdot 45 \pi}\right] \text { with }-0.45 \pi \leqslant X \leqslant 0.45 \pi, \\
y=\frac{1}{2}\left[1+\frac{\sin Y}{\sin 0 \cdot 45 \pi}\right] \text { with }-0.45 \pi \leqslant Y \leqslant 0.45 \pi,
\end{gathered}
$$

with 21 equally spaced grid points in each co-ordinate direction ( $X$ and $Y$ ) in the transformed plane. For $A \leqslant 0 \cdot 2$, the transformation (5) required far too many grid points in the $X$ direction to provide the necessary resolution in the end regions. Hence, while the transformation (6) was retained for the vertical co-ordinate, we resorted to what we shall refer to as an arbitrarily discretized grid for the horizontal direction in these cases. With this approach, the discrete step size $\Delta x_{i}$ was chosen to be a function of the co-ordinate position $i$. Although the spacing of the arbitrary grid was different for each case studied, the choice was made in a consistent manner, based primarily on the observation from I that the overall flow is composed of a core region in which the horizontal derivatives scale as $1 / A$ and two end regions whose extent is independent of $A$ (for $A \ll 1$ ). The same resolution of the fluid motion in each of these three regions was obtained by splitting the grid points up evenly with one third of the points in each region. With the final requirement that the grid spacing vary smoothly 
from a minimum near the end walls to a maximum in the core, an adequate grid was obtained using a $21 \times 45$ mesh system.

Once converged solutions had been obtained on the $21 \times 45$ grid, they were recomputed using a $21 \times 55$ grid, the extra ten points being split evenly between the two end regions. In all cases, the new values of the stream function differed from the results using the coarser grid only in the fourth significant figure. Furthermore, the integral properties, such as the overall Nusselt number, differed by $1 \%$ or less. The addition of grid points in the core had no effect on the final solution. This degree of consistency was deemed acceptable in view of the very large increase in computer costs required to obtain the minor benefits of any further increase in accuracy. As we shall see, a quantitative indication of the error introduced by this finite-difference scheme is provided by comparison of the numerical results with the asymptotic theory of $I$.

The difference equations which result from either the transformation (5) and (6) or the transformation (6) with the arbitrarily discretized horizontal grid may be expressed in a similar form and were derived in the present work by replacing time derivatives in (1) and (2) with forward differences, by representing the Jacobian (or convective) terms in the conservative form suggested by Arakawa (1966) and by replacing all other spatial derivatives with two-point central differences. The general form of the difference equations which result is well known and hence, in the interest of brevity, we shall not repeat them here $\dagger$.

To incorporate the boundary conditions into the difference scheme, we have transformed the two boundary conditions on $\psi$ to an explicit representation for both $\psi$ and $\omega$ on the cavity walls. An appropriate relationship for $\omega$, accurate to $O(\Delta x)^{2}$ or $O(\Delta y)^{2}$, may be obtained from Taylor series expansions of $\psi$ at the internal rows or columns nearest the wall, combined with the boundary conditions (4) and equation (2) evaluated at the boundary.

\section{The numerical algorithm}

Although we were interested only in the steady-state solution, we chose to integrate the transient difference equations from an initial guess at the steady flow configuration to the final steady state by means of the two-step, alternating direction, implicit method (A.D.I.) developed by Peaceman \& Rachford (1955). The systems of tridiagonal linear equations that resulted at each step of the integration were solved by means of the Thomas algorithm (Von Rosenberg 1969 , p. 113). The A.D.I. technique has the advantage over explicit methods that it is numerically more stable and hence allows the use of a larger time step $\Delta t$. It has the disadvantage, however, that each iteration requires more computation than does an iteration with the explicit techniques.

We found also that a larger discrete time step could be used for the integration of the temperature equation than could be used for the vorticity equation. Hence, to reduce the computation time required to reach the final solution, a different time step was used in each equation. For the cases which we investigated, the

$\uparrow$ A copy of these may be obtained from the authors, or the editor, upon request. 


\begin{tabular}{|c|c|c|c|c|c|c|c|}
\hline Case & $G r$ & $A$ & $\operatorname{Pr}$ & $G r^{2} A^{3}$ & $\mathrm{Nu}$ & $K_{1}$ & $\psi_{\max }$ \\
\hline I & $2 \times 10^{4}$ & $1 \cdot 0$ & 0.733 & - & $2 \cdot 64$ & - & $3.92 \times 10^{-4}$ \\
\hline II & $2 \times 10^{4}$ & $1 \cdot 0$ & 6.983 & $4 \times 10^{8}$ & $5 \cdot 55$ & - & $8.91 \times 10^{-5}$ \\
\hline III & $2 \times 10^{4}$ & $0 \cdot 2$ & 6.983 & $3.2 \times 10^{6}$ & $4 \cdot 07$ & $0 \cdot 166$ & $5.16 \times 10^{-4}$ \\
\hline IV & $2 \times 10^{4}$ & $0 \cdot 1$ & $6 \cdot 983$ & $4 \times 10^{5}$ & $3 \cdot 10$ & 0.336 & $1.01 \times 10^{-3}$ \\
\hline $\mathrm{V}$ & $2 \times 10^{4}$ & 0.05 & 6.983 & $5 \times 10^{4}$ & 1.68 & 0.603 & $1.65 \times 10^{-3}$ \\
\hline VI & $2 \times 10^{3}$ & $0 \cdot 1$ & 6.983 & $4 \times 10^{3}$ & 0.417 & 0.840 & $2 \cdot 33 \times 10^{-3}$ \\
\hline VII & 500 & $0 \cdot 1$ & $6 \cdot 983$ & $2 \cdot 5 \times 10^{2}$ & $0 \cdot 130$ & 0.968 & $2.70 \times 10^{-3}$ \\
\hline VIII & 10 & $0 \cdot 1$ & $6 \cdot 983$ & $0 \cdot 1$ & $0 \cdot 1006$ & 0.996 & $2.75 \times 10^{-3}$ \\
\hline
\end{tabular}

temperature time step was chosen as much as 1000 times larger than the vorticity time step.

Computation time was further minimized by making use of the centro-symmetry property of the combined equations and boundary conditions. In particular, if there are $n_{x}$ grid points in the $x$ direction and $n_{y}$ grid points in the $y$ direction, then symmetry requires

$$
\left.\begin{array}{rl}
\psi(i, j) & =\psi\left(n_{x}-i, n_{y}-j\right), \\
\omega(i, j) & =\omega\left(n_{x}-i, n_{y}-j\right), \\
\theta(i, j) & =\mathbf{1}-\theta\left(n_{x}-i, n_{y}-j\right) .
\end{array}\right\}
$$

Hence, only half of the grid points need be scanned at each iteration, leading to at least a $50 \%$ cut in computation time.

Excellent indications of the progress of the integration were provided by an integral of the vorticity over one end of the cavity (essentially, a drag coefficient) and by the Nusselt number as defined in equation (I48). Once the variations in both of these integral parameters between iterations were of the same magnitude as the computer truncation error, the integration was stopped. For the IBM 370-155 which was used, this occurred when changes were taking place in the fifth or sixth significant figure. Typical computation times using the approach outlined above were $20-30 \mathrm{~min}$ with the variations due primarily to changes in the Grashof number and to variations in the accuracy of the starting values assumed for $\theta, \psi$ and $\omega$.

\section{Numerical results}

\section{Square box}

As a preliminary test to establish the overall consistency of the numerical results produced by our algorithm, we considered a square cavity with

$$
G r=2 \times 10^{4} \text { and } \operatorname{Pr}=0 \cdot 733,
$$

listed as case I in table 1 . Wilkes \& Churchill (1966) had previously considered the same system. With a $21 \times 21$ grid in the Cartesian co-ordinate system, they obtained a Nusselt number of $2 \cdot 52$, whereas using (5) and (6), with 21 points in each o-ordinate direction, we obtained $N u=2 \cdot 64$. We consider the difference 


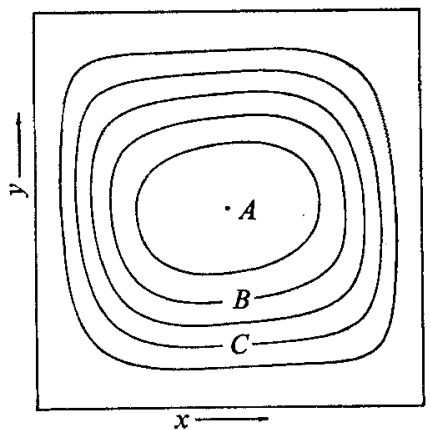

(a)

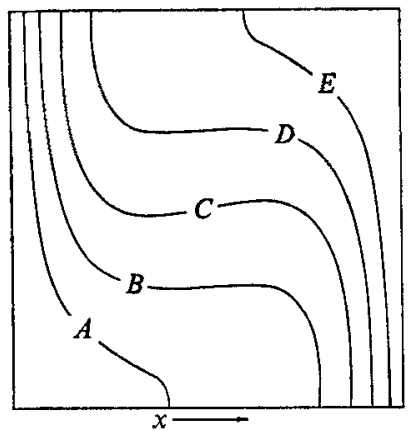

(b)

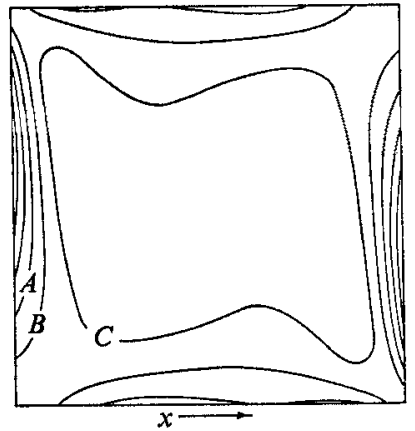

(c)

Figure 1. Case I; $G r=2 \times 10^{4}, A=1, P r=0.733$. (a) Streamlines; $A=3.92 \times 10^{-4}$, $B=2.62 \times 10^{-4}, C=1.31 \times 10^{-4}$. (b) Isotherms; $A=0.167, B=0.333, C=0.5$, $D=0.667, E=0.833$. (c) Vorticity contours; $A=-1.41 \times 10^{-2}, B=-5.77 \times 10^{-3}$, $C=2.55 \times 10^{-3}$.

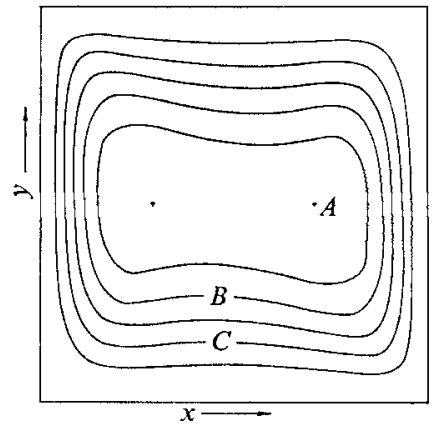

(a)

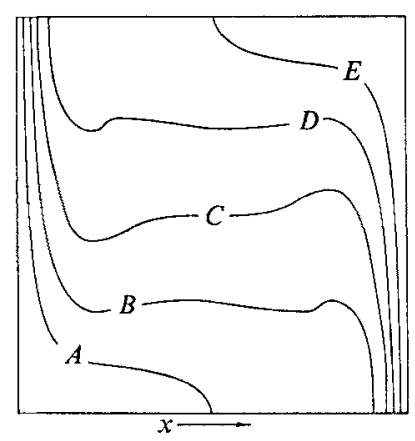

(b)

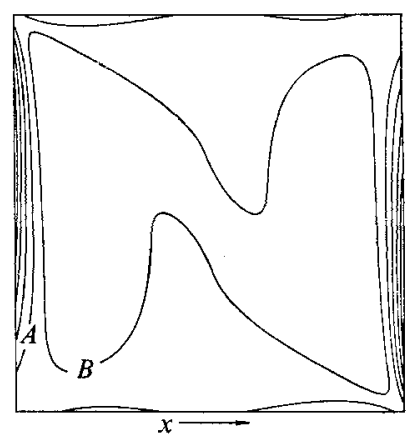

(c)

Figure 2. Case II; $G r=2 \times 10^{4}, A=1, P r=6.983$. (a) Streamlines; $A=8.91 \times 10^{-4}$, $B=5.90 \times 10^{-4}, C=2.96 \times 10^{-4}$. (b) Isotherms; $A=0.167, B=0.333, C=0.5$, $D=0.667, E=0.833$. (c) Vorticity contours; $A=-4.24 \times 10^{-3}, B=7.6 \times 10^{-4}$.

between these values to be largely insignificant, particularly in view of the large scatter of the numerically determined values for $N u$ as a function of $R a$ that have been collected by Quon (1972) from various authors (cf. his figure 9).

In figure 1 the flow configuration is presented for this case. The structure of the isotherms indicates that the only substantial horizontal temperature gradients occur in the lower right and upper left ends of the cavity. Since the buoyancy forces are proportional to the temperature gradients [cf. equation (1)], the overall flow must be driven by these essentially boundary-layer-like regions, with the flow in the core maintained by the requirements of continuity as suggested by the model of Gill (1966).

A change in the Prandtl number from 0.733 to 6.983 (the Prandtl number of water at $20^{\circ} \mathrm{C}$ ) with all other parameters held constant produced a considerable change in flow structure, as indicated by comparing figures 1 and 2 . The chief change is a thinning of the thermal boundary layer, which is to be expected 
on the basis of standard boundary-layer arguments (Gill 1966). Furthermore, since it is the buoyancy-induced forces in the thermal boundary-layer region which drive the flow, the region of largest vertical velocity is also confined more closely to the walls, as is evident in figure $2(a)$.

A secondary effect of increasing $P r$ is a substantial decrease in the maximum value of the stream function within the cavity. $\dagger$ Furthermore, a secondary flow develops which may be recognized in figure 2 by the two stream-function maxima. This induced secondary flow not only causes inflexions in the isotherms, but also convects the negative vorticity that is generated in the vicinity of the walls further into the bulk of the fluid. These secondary flow phenomena are consistent both with the numerical results of De Vahl Davis (1968), Rubel \& Landis (1969) and Quon (1972) and with the experimental results of Elder (1965), who found that such a flow results for $R a>10^{5}$. For the present case $R a=1 \cdot 4 \times 10^{5}$. This strong dependence of the flow characteristics on $\operatorname{Pr}$ for $A=1$ is in contrast to the conclusion in $I$ that the stream-function and vorticity fields should be independent of $\operatorname{Pr}$ to first order for $A \ll 1$.

\section{Cavities with $A<1$}

It was pointed out in I [equation (I51)], that, for constant $P r$, a significant parameter in determining whether the flow in the core complies with the parallel flow structure derived in $\mathrm{I}$ is $G r^{2} A^{3}$. To illustrate the transition from the boundarylayer regime of large $G r^{2} A^{3}$ to the parallel flow structure of 'small' $G r^{2} A$, we have carried out computations at several values of this parameter. The cases considered are shown in table 1 , in order of decreasing $G r^{2} A^{3}$. Also listed are the numerically calculated Nusselt numbers and maximum stream function $\psi_{\max }$ for each case.

All of the results for $A<1$ deal only with $\operatorname{Pr}=6.983$, primarily so that the results can be directly compared with experimental observations in water (see part 3), but also to take advantage of the observation of De Vahl Davis (1968) that the stability of the numerical algorithm increases with increasing $P r$.

In figures 3-8, we have plotted the streamlines, isotherms and contours of constant vorticity for each case, also arranged in order of decreasing $G r^{2} A^{3}$ so that the trends of the numerical solution can easily be identified. It should be noted that all of the results are plotted as square figures; hence, for cavities with small aspect ratios the horizontal length scale is substantially compressed.

An examination of the results for the cavities with $G r=2 \times 10^{4}, \operatorname{Pr}=6.983$ and various aspect ratios (figures $2-5$ ) reveals that the streamlines and vorticity lines become more nearly parallel as $A$ decreases, as was anticipated in the analysis of $\mathrm{I}$. It is perhaps worth emphasizing that this transition to parallel flow is not simply a consequence of decreasing the overall temperature gradient $\left(T_{h}-T_{c}\right) / l$, as the aspect ratio is made smaller. In fact, one can change $A$ in such a manner that the overall temperature difference must actually be increased in

The apparent difference between this result and that of De VahI Davis (1968) is due both to different scaling and to the fact that we held $G r$ fixed while De Vahl Davis used $R a(=G r P r)$ as the fixed parameter. 


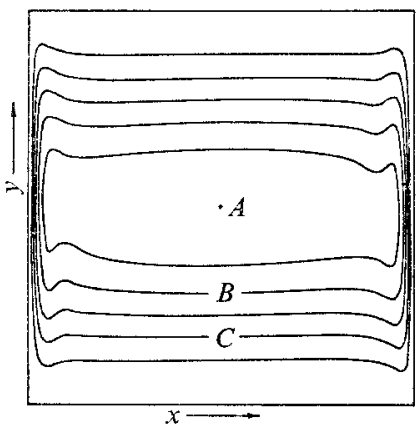

(a)

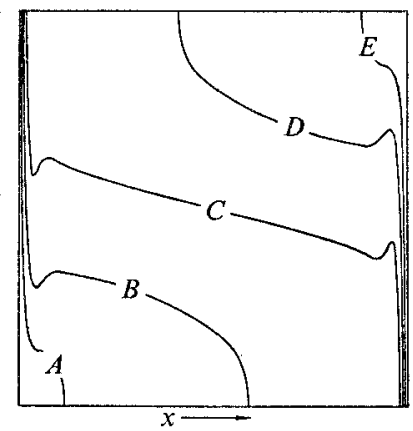

(b)

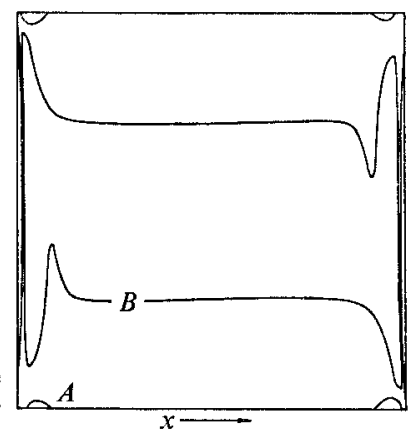

(c)

Figure 3. Case III; $G r=2 \times 10^{4}, A=0 \cdot 2, P r=6 \cdot 983$. (a) Streamlines; $A=5 \cdot 16 \times 10^{-4}$, $B=3.45 \times 10^{-4}, C=1.73 \times 10^{-4}$. (b) Isotherms; $A=0.167, B=0.333, C=0.5$, $D=0 \cdot 667, E=0.833$. (c) Vorticity contours; $A=-1.88 \times 10^{-2}, B=3.48 \times 10^{-3}$.

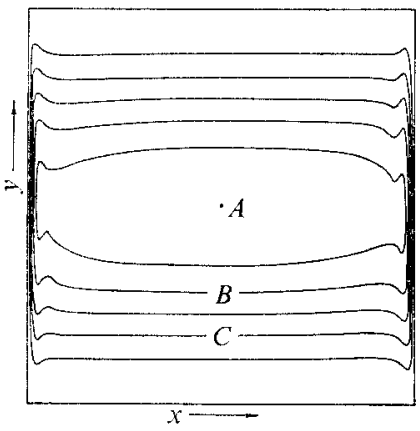

(a)

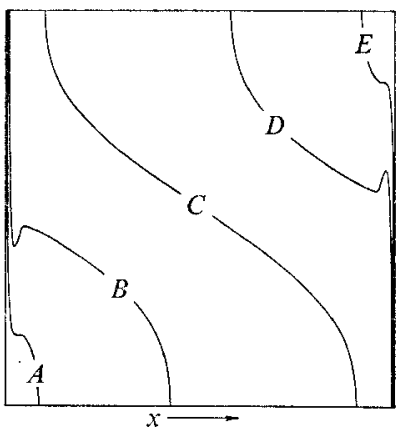

(b)

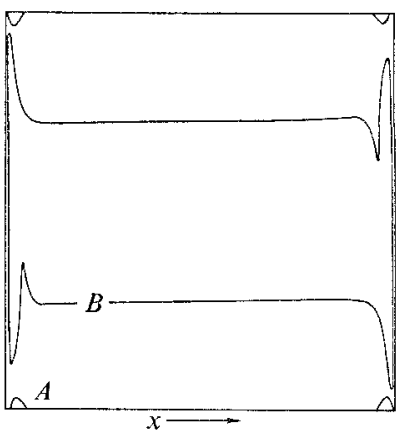

(c)

Figure 4. Case IV; $G r=2 \times 10^{4}, A=0 \cdot 1, P r=6 \cdot 983$. (a) Streamlines; $A=1 \cdot 01 \times 10^{-3}$, $B=6.72 \times 10^{-4}, C=3.36 \times 10^{-4}$. (b) Isotherms; $A=0.167, B=0.333, C=0.5$, $D=0 \cdot 667, E=0 \cdot 833$. (c) Vorticity contours; $A=-3.18 \times 10^{-2}, B=6 \cdot 28 \times 10^{-3}$.

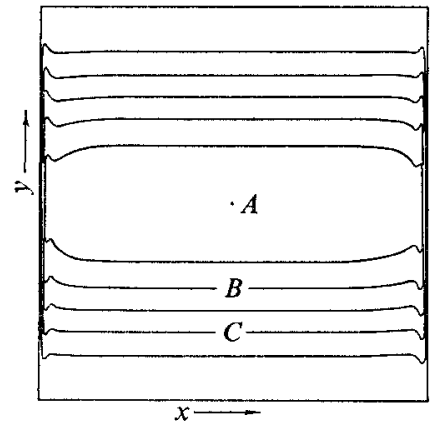

(a)

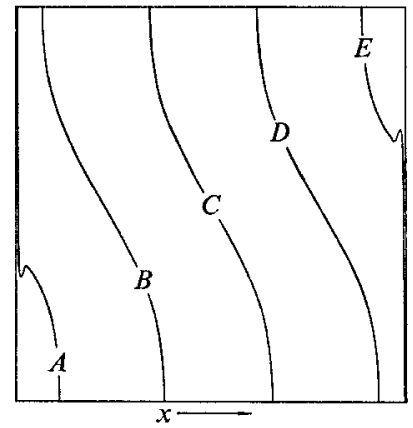

(b)

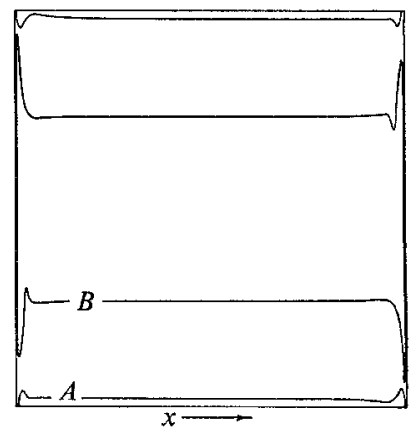

(c)

Figure 5. Case V; $G r=2 \times 10^{4}, A=0.05, P r=6.983$. (a) Streamlines; $A=1.65 \times 10^{-3}$, $B=1.11 \times 10^{-3}, C=5.53 \times 10^{-4}$. (b) Isotherms; $A=0.167, B=0.333, \quad C=0.5$, $D=0.667, E=0.833$. (c) Vorticity contours; $A=-4.43 \times 10^{-2}, B=9.09 \times 10^{-3}$. 


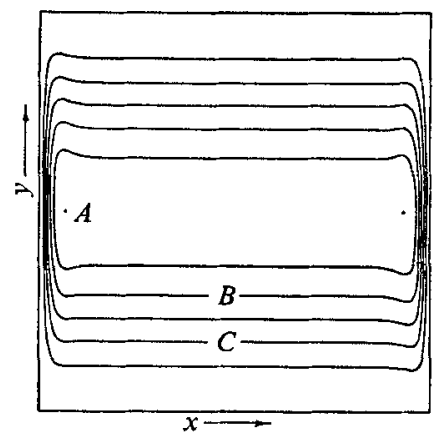

(a)

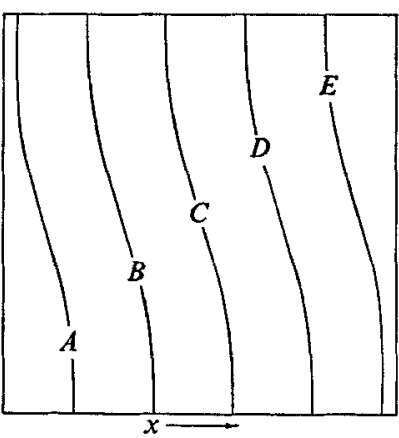

(b)

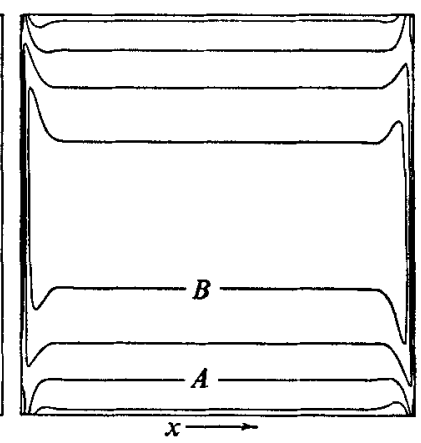

(c)

Figure 6. Case VI; $G r=2 \times 10^{3}, A=0.1, P r=6.983$. (a) Streamlines; $A=2.33 \times 10^{-3}$, $B=1.55 \times 10^{-3}, C=7.74 \times 10^{-4}$. (b) Isotherms; $A=0.167, B=0.333, C=0.5$, $D=0.667, E=0.833$. (c) Vorticity contours; $A=-2.60 \times 10^{-1}, B=2.20 \times 10^{-2}$.

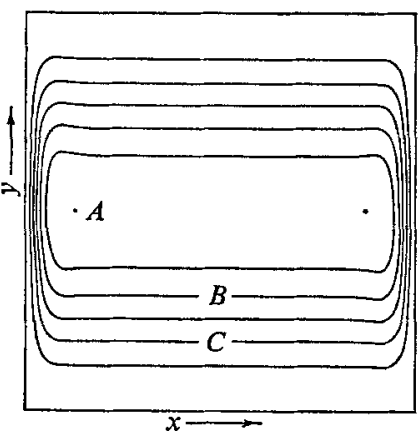

(a)

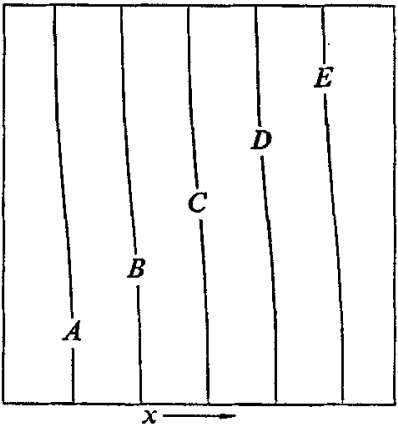

(b)

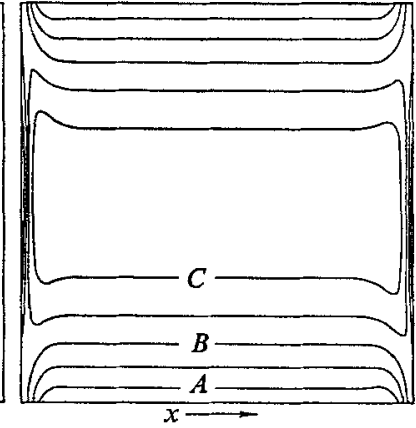

(c)

FIgURe 7. Case VII; $G r=500, A=0 \cdot 1, P r=6 \cdot 983$. (a) Streamlines; $A=2 \cdot 70 \times 10^{-3}$, $B=1.80 \times 10^{-3}, C=8.99 \times 10^{-4}$. (b) Isotherms; $A=0.167, B=0.333, C=0.5$, $D=0.667, E=0.833$. (c) Vorticity contours; $A=-6.31 \times 10^{-2}, B=-1.96 \times 10^{-2}$, $C=2 \cdot 40 \times 10^{-2}$

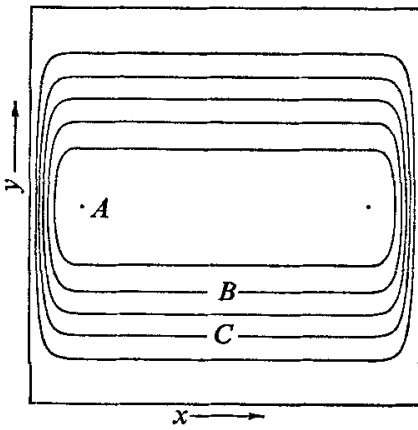

(a)

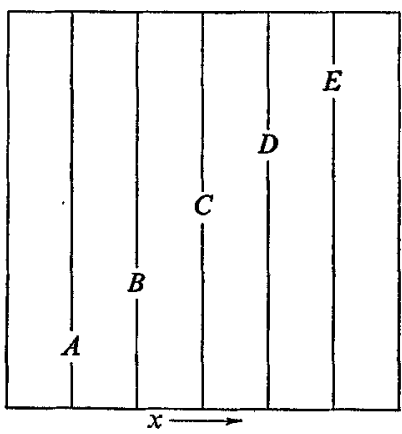

(b)

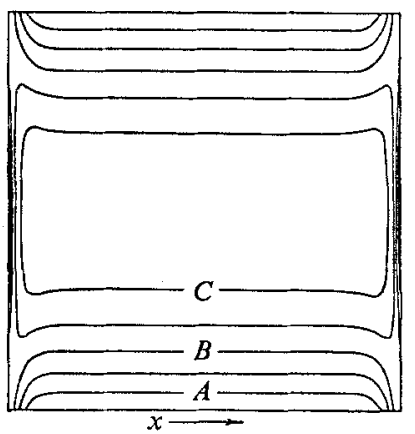

(c)

Figure 8. Case VIII; $G r=10, A=0 \cdot 1, P r=6.983$. (a) Streamlines; $A=2.75 \times 10^{-3}$, $B=1.84 \times 10^{-3}, C=9.17 \times 10^{-4}$. (b) Isotherms; $A=0.167, B=0.333, C=0.5$, $D=0.667, E=0.833$. (c) Vorticity contours; $A=-6.26 \times 10^{-2}, B=-1.99 \times 10^{-2}$, $C=2.28 \times 10^{-2}$. 
order to hold $G r$ fixed. For example, $A$ may be decreased by simply decreasing $h$ while holding $l$ constant. Then, to maintain a specified constant

$$
G r\left(\equiv \beta g\left(T_{h}-T_{c}\right) h^{3} / \nu^{2}\right),
$$

it is obviously necessary to increase $\left(T_{h}-T_{c}\right) / l$.

In addition to the increasingly parallel character of the flow, the gradual disappearance of the thermal boundary layers as $A$ is decreased is also a prominent feature of the transition. In case II, the core flow is driven primarily by the entrainment and detrainment of fluid from the buoyancy-driven boundary layers. On the other hand, by case $\mathrm{V}$ these boundary layers have essentially disappeared. The major portion of the temperature drop occurs across the core, so that the overall flow is necessarily driven by the buoyancy forces in the core. This transition to a core-driven flow, with decreasing $A$, was suggested in I and is completely consistent with the asymptotic theory.

The above tendencies are not restricted to the situation with $G r$ fixed and $A$ decreasing. A consideration of the figures in order of decreasing $G r$ with $A$ fixed (figures 4, 6, 7 and 8) reveals a similar trend of increasingly parallel flow and a transition from a thermal boundary-layer structure to a linear temperature profile. This behaviour provides support for the qualitative conclusion from I that the condition (I51) is sufficient for the existence of the parallel core flow.

Figures 3-8, when observed in order of decreasing $G r^{2} A^{3}$, yield some additional tendencies, most of which fall within the scope of the asymptotic analysis. Of particular prominence is the decreasing magnitude of the stream-function deflexions that occur as the fluid enters and leaves the end regions. A prediction from the asymptotic theory was that these deflexions, for constant $P r$, should be $O\left(G r^{2} A^{2}\right)$, provided that $A$ is sufficiently small. Since the arrangement in order of decreasing $G r^{2} A^{3}$ also produces an arrangement in order of decreasing $G r A$, this phenomenon is again compatible with the asymptotic theory.

One characteristic of the stream-function deflexions at the larger values of $G r A$ which is not predicted by the first four terms of the asymptotic expansion is the 'necking down' of the streamlines before they again diverge. $\dagger$ Closely associated with the necking phenomenon is the occurrence of the stream-function maximum in the core rather than in the end regions as predicted in the asymptotic theory. Only in cases VI-VIII, for which necking is absent, does the absolute maximum of $\psi$ occur in the end regions. However, a closer examination of the numerical results does indicate that there always exists at least a local maximum of $\psi / r$ in each end region.

Some additional interesting trends are also displayed by the plots of the isotherms and equi-vorticity lines. For the larger values of $G r^{2} A^{3}$, the vorticity gradients are extremely large in the vicinity of the end walls, and, in relative terms, are almost non-existent in the bulk of the fluid. Hence, in this limited sense, Batchelor's (1954) conjecture of a uniform-vorticity core at large $\operatorname{Gr}$ ( $A$ fixed, $G r \rightarrow \infty)$ is basically confirmed. It is important to note, however, that the basic

$\uparrow$ There is, however, a strong indication that this phenomenon would be realized with a subsequent (higher-order) correction to the solution in the end region. 
flow structure in this limit is otherwise completely unlike that suggested by Batchelor and in qualitative accord with that proposed by Gill. In particular, because of the strong horizontal advection due to entrainment-detrainment from the boundary layers, the temperature distribution in the core at large $G r^{2} A^{3}$ shows only a weak dependence on horizontal position, while still retaining reasonably strong vertical gradients. The vorticity generated at the end walls does not effectively diffuse to the core, because the buoyancy forces in the region adjacent to the walls provide an effective sink for this vorticity (they appear as source terms for vorticity of opposite sign) which greatly decreases its magnitude before it can reach the core. A lucid explanation of the transient development of this (large $G r^{2} A^{3}$ ) physical structure has recently been provided by Quon (1972). As either $A$ or $G r$ is decreased, the magnitude of the vorticity gradients in the end regions is decreased, and the temperature distribution shows a gradual transition from the strong vertical dependence of the boundary-layer regime towards the totally horizontal dependence corresponding to the linear profile of I. Ultimately, in cases VI-VIII, the vorticity gradients are of comparable magnitude throughout the cavity. In addition, as expected from the asymptotic analysis, the vorticity distribution becomes increasingly symmetric with decreasing $A$ or $G r$.

A more quantitative comparison of the numerical solutions with the asymptotic theory is possible particularly for the flow in the core. Equation (I14) indicates that, if the flow is parallel, then

$$
u / K_{1}=\partial^{2} f(y) / \partial y^{2}
$$

where

$$
f(y)=\frac{1}{120} y^{5}-\frac{1}{48} y^{4}+\frac{1}{72} y^{3}
$$

and $K_{1}$ is the parallel-flow parameter. Any deviation of numerically determined values of $u / K_{1}$ from the relationship in (8) will give a good indication of deviations of the numerical solutions from the parallel flow structure. Hence, the problem of quantitatively comparing the numerical and analytical velocity profiles reduces to one of finding a value for $K_{1}$ from each numerical solution. This is easily done since, according to equation (I15), the core temperature can be separated into two parts when $A$ is sufficiently small. One part is a linear function of $x$ and the other is the fifth-order polynomial $f(y)$. Hence, a value for $K_{1}$ can be determined from each numerical solution (whether or not the flow is identically parallel in the core) by fitting the numerically determined temperature $\theta_{i j}$, in a least-squares manner, to the equation

$$
\theta=K_{1} A x+K_{2}
$$

at the five central grid points, on the line $y=0.5$. The resulting values of $K_{1}$ are presented in table 1. As expected in view of the transition from the boundarylayer regime to the 'conduction' regime of the $\mathrm{I}$, the coefficient $K_{1}$ increases as $G r^{2} A^{3}$ decreases.

Figure 9 is a plot of the theoretically and numerically determined velocity profiles $u / K_{1}$ on the centre-line $x=1 / 2 A$ as a function of $y$. It is apparent that as $G r^{2} A^{3}$ decreases the velocity profile approaches more elosely the parallel structure. However, for values of $G r^{2} A^{3}<10^{4}$, cases VI-VIII, the numerical 


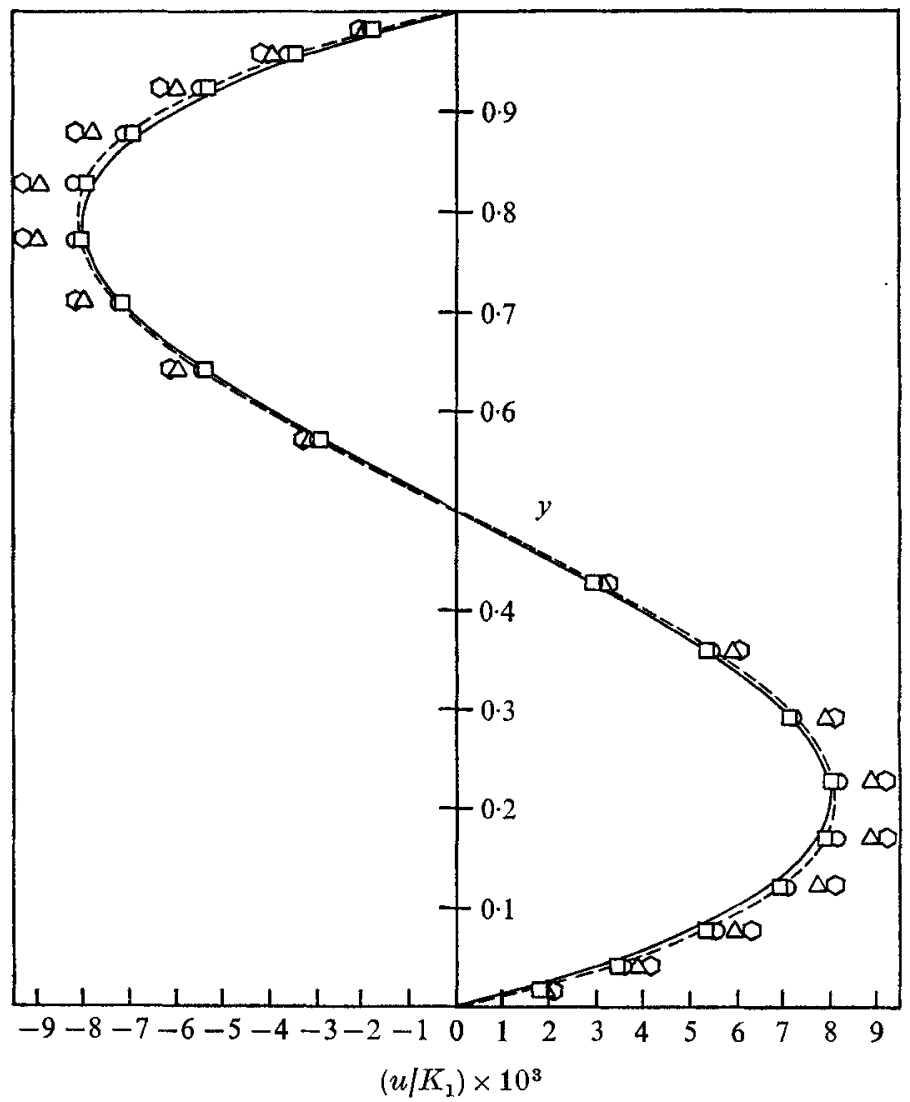

FIGURE 9. Comparison of normalized horizontal velocity at $x=1 / 2 A$ with theoretical parallel-flow profile (solid line). $\bigcirc, G r=2 \times 10^{4}, A=0.2 ; \triangle, G r=2 \times 10^{4}, A=0.1$; $\bigcirc, G r=2 \times 10^{4}, A=0.05 ; \square, G r=2 \times 10^{3}, A=0.1 ;---, G r=5 \times 10^{2}, A=0 \cdot 1$.

data fall on a single curve (the broken line) that is somewhat different from the theoretical profile. Because of its consistency, we attribute this discrepancy to the numerical error introduced by the finite-difference scheme and grid network used. Since this error constitutes only a very small fraction of $\hat{u} / K_{1}$, the correspondence between the analytical theory and the limiting form of the numerical velocity profiles would appear to be quite satisfactory.

It is significant that cases III-V, in which $G r^{2} A^{3}$ is largest, have horizontal velocities larger than those predicted by the parallel-flow theory with $K_{1}$ evaluated from the numerical solutions. As the transition from the asymptotic limit of I to the boundary-layer regime of Gill is encountered, the driving force for fluid motion must gradually change over from one totally dominated by the horizontal temperature gradient in the core to one controlled by entrainmentdetrainment from the boundary layers at the end walls. Hence, as $G r^{2} A^{3}$ is increased, the theoretical prediction from I must increasingly underestimate the actual horizontal velocities, since it is based entirely on the core-gradient mechanism. This is evident in the results of figure 9. 


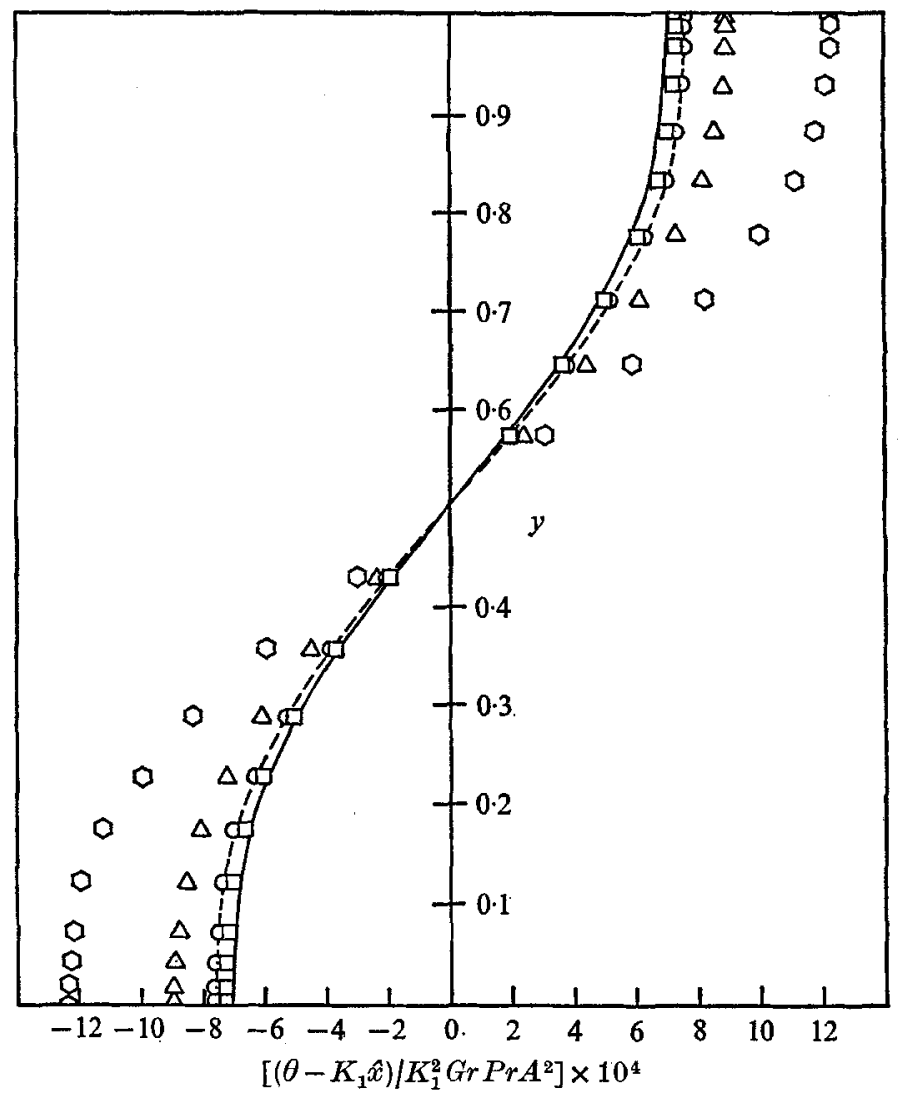

FIGURE 10. Comparison of numerically determined $\theta$ at $x=1 / 2 A$ with theoretical core temperature (solid line). Notation as in figure 9.

It is also possible to obtain a quantitative estimate for the value of $G r^{2} \operatorname{Pr}^{2} A^{3}$ at which the core flow changes from parallel to non-parallel from the results presented in figure 9, and hence to verify condition (I51). The velocity profile of case $V$ deviates only slightly from the limiting profile of the numerical solutions, whereas that for case VI falls directly on the limiting curve. Hence, the appropriate limit for validity of the parallel flow structure (for $A \sim 0 \cdot 1$ ) must occur at a $G r^{2} \mathrm{Pr}^{2} A^{3}$ between $2.5 \times 10^{6}$ (case V) and $2 \times 10^{5}$ (case VI). This observation lends strong support to the speculation of $I$ that the parallel flow structure will apply for $G r^{2} \operatorname{Pr}^{2} A^{3} \lesssim 10^{5}$, provided that $A$ is sufficiently small.

Further consideration of the core solution of $I$ indicates that a plot of

$$
\left(\theta-K_{1} A x\right) / K_{1}^{2} G r P r A^{2}
$$

as a function of $y$ should also yield the fifth-order polynomial $f(y)$, provided that $A$ and $G r^{2} \operatorname{Pr}^{2} A^{3}$ are such that the parallel-flow theory is relevant. Figure 10 is a plot of this function evaluated at the centre-line $x=1 / 2 A$. For cases IIIVI, with decreasing $G r^{2} A^{3}$, the numerical profiles approach the theoretical curve as would be expected. On the other hand, for case VII the difference between the 
numerical and theoretical curves increases slightly and for case VIII, which is not shown on the figure, the differences between the theoretical and numerical data points are large and random. Although this latter trend would, at first glance, appear to be inconsistent with the theory of $I$, it is in fact a numerical artifact which is a consequence of the structure of the temperature field as $\operatorname{Pr} \operatorname{Gr} A^{2} \rightarrow 0$. An order-of-magnitude analysis of equation (I15) reveals that both $K_{1} A x$ and $\theta$ are $O(1)$ whereas

$$
K_{1}^{2} G r \operatorname{Pr} A^{2} f(y)=O\left(G r \operatorname{Pr} A^{2} \times 10^{-3}\right) .
$$

If we assume, for some hypothetical values of the parameters $G r, \operatorname{Pr}$ and $A$, that the core flow is identically parallel and, furthermore, that the numerically determined temperature field contains a consistent error $\xi_{1}$, then a simple calculation shows that the error in the numerical data corresponding to figure 10 will be amplified to

To be specific, for case VII,

$$
\xi_{2}=\xi_{1} / \operatorname{Gr} \operatorname{Pr} A^{2} \times 10^{-3}
$$

$$
G r \operatorname{Pr} A^{2} \times 10^{-3}=0.035
$$

so that, if the numerically determined temperature profile contains an error of $0.5 \%$, then it is not unreasonable to have an error of $15 \%$ in the reduced numerical data. Similarly, for case VIII,

$$
\operatorname{Gr} \operatorname{Pr} A^{2} \times 10^{-3}=7 \times 10^{-4} \text {, }
$$

so that even a small numerical error would be amplified to substantial proportions. We must conclude that, owing to the increasing prominence of even the smallest numerical errors as $\operatorname{Gr} \operatorname{Pr} A^{2}$ decreases, figure 10 is of limited value in establishing the region of $G r, \operatorname{Pr}, A$ space in which the core-flow solution is valid.

In the previous discussion, it has been emphasized that the numerical results effectively display the transition from the parallel flow regime of small $G r^{2} A^{3}$ to the boundary-layer regime of large $G r^{2} A^{3}$. Perhaps the most graphic demonstration of this transition is provided by figure 11 , a plot of $\log \left(1-K_{1}\right)$ as a function of $\log \left(G r^{2} A^{3}\right)$. The asymptotic expression for $K_{1}$, equation (145), indicates that for constant $\operatorname{Pr}$ such a plot should yield a curve that is asymptotic to the straight line

$$
\log \left(1-K_{1}\right)=\log \left(3 \cdot 48 \times 10^{-6} \operatorname{Pr}^{2}\right)+\log \left(G r^{2} A^{3}\right)
$$

in the limit as $A \rightarrow 0$. On the other hand, the boundary-layer theory suggests that for large $G r^{2} A^{3}$ the horizontal gradient of temperature in the core should be zero. Hence for large $G r^{2} A^{3}$ the eurve should be asymptotic to the line

$$
1-K_{1}=1 \text {. }
$$

The numerically determined values of $K_{1}$ display the expected trends in both limits.

A further demonstration of the transition from the parallel to the boundarylayer regime is provided by figure 12, a plot of $\log (N u-A)$ as a function of $\log \left(G r^{2} A^{3}\right)$. We emphasize that it is the deviation of the actual $N u$ from the asymptotic value $A$ that is plotted here. For small values of $G r^{2} A^{3}$, the 
Convection in a shallow cavity with heated walls. Part 2

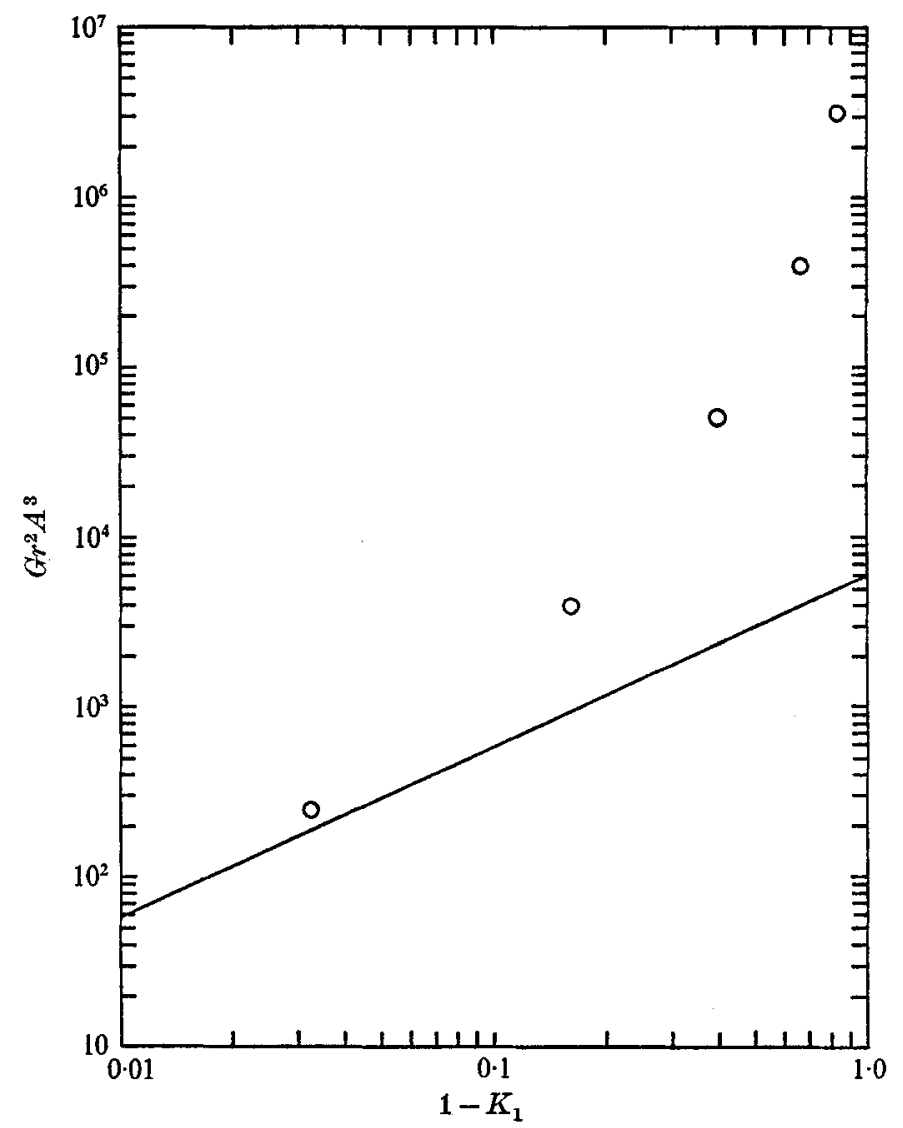

FIGURE 11. Variation of $K_{1}$ with $G r^{2} A^{3} .0$, numerical data; - asymptotic theory.

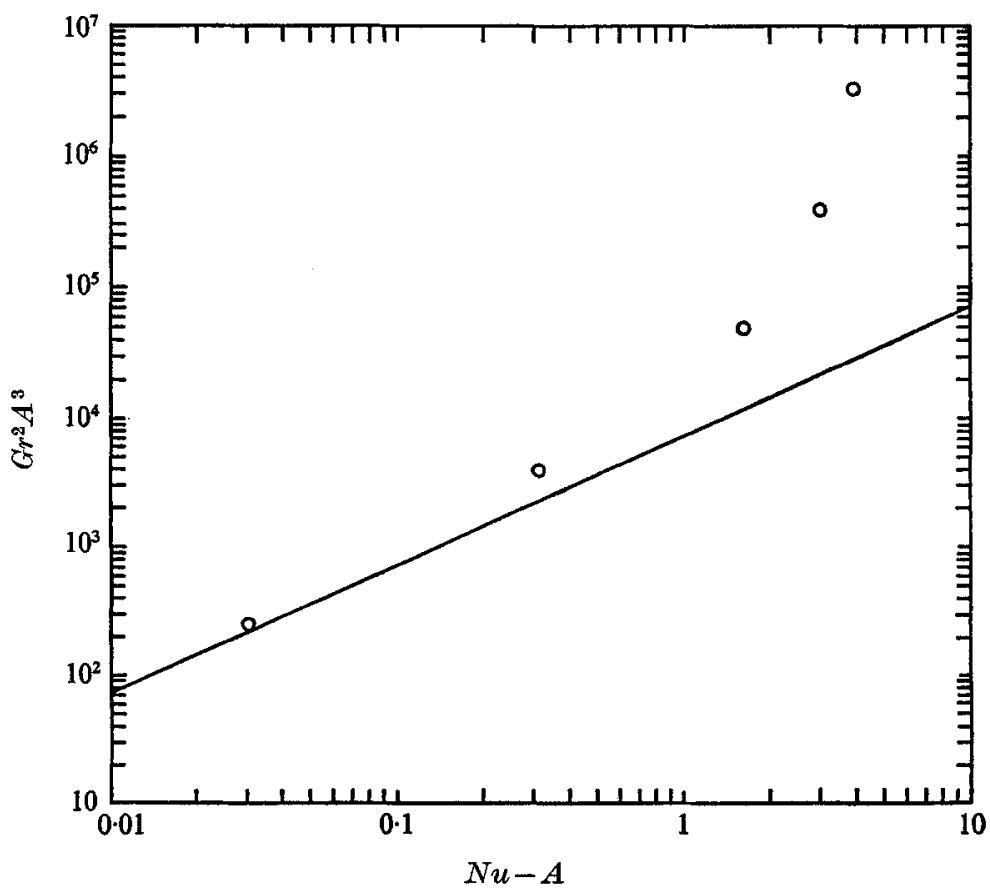

FraURe 12. Variation of $N u-A$ with $G r^{2} A^{3} . \bigcirc$, numerical data; - asymptotic theory. 
numerically determined values of the Nusselt number are asymptotic to the theoretical straight line which was derived in I. On the other hand, it is also evident that, as $G r^{2} A^{3}$ increases, the numerically determined Nusselt numbers deviate considerably from the asymptotic value. In fact, for the largest values of $G r^{2} A^{3}$ considered, the scaling of figure 12 apparently has little bearing on the actual Nusselt number. This is to be expected, however, since at large values of $G r^{2} A^{3}$ the flow resembles more closely the boundary-layer structure than it does the parallel flow structure. Since Gill's boundary-layer analysis showed that $N u$ should be independent of $A$, and vary directly as $(G r P r)^{\frac{1}{2}}$, the sharp increase in the slope of the numerical curve at large values of $G r^{2} A^{3}$ can be attributed to the transition to the boundary-layer structure.

This work was supported, in part, by National Science Foundation Grant GK-35476.

\section{REFERENCES}

Arakawa, A. 1966 J. Comp. Phys. 1, 119.

Batchelor, G. K. 1954 Quart. Appl. Math. 12, 209.

Cormack, D. E., Leat, L. G. \& Imberger, J. 1974 J. Fluid Mech. 65, 209.

De VaHL Davis, G. 1968 Int. J. Heat Mass Transfer, 11, 1675.

ELDER, J. W. 1965 J. Fluid Mech. 23, 77.

ELDER, J. W. 1966 J. Fluid Mech. 24, 823.

GILL, A. E. 1966 J. Fluid Mech. 26, 515.

NeWELL, M. E. \& SCHMIDT, F. W. 1970 J. Heat Transfer, $92,159$.

Peaceman, D. W. \& Rachford, H. H. 1955 J. Soc. Ind. Appl. Math. 3, 28.

Quon, C. 1972 Phys. Fluids, 15, 12.

Rubet, A. \& Landis, F. 1969 Phys. Fluids Suppl. II, 12, II-208.

Von Rosenberg, D. U. 1969 Methods for the Numerical Solution of Partial Differential Equations. Elsevier.

Wilkes, J. O. \& ChURChILL, S. W. 1966 A.I.Ch.E. J. 12, 161. 